\title{
Effects of Glenoid and Humeral Bone Defects on Recurrent Anterior Instability of the Shoulder
}

\author{
In Park, MD, Min-Joon Oh, MD*, Sang-Jin Shin, MD \\ Department of Orthopedic Surgery, Ewha Womans University Seoul Hospital, Ewha Womans University School of Medicine, Seoul, \\ *Department of Orthopedic Surgery, Ewha Womans University Mokdong Hospital, Ewha Womans University School of Medicine, Seoul, Korea
}

For proper treatment of recurrent anterior instability of the shoulder with a bone defect, the defect size should be assessed preoperatively with three-dimensional computed tomography or magnetic resonance imaging. In general, the risk of postoperative recurrence of instability is estimated on the basis of preoperative imaging of bipolar bone defects: more than $20 \%-25 \%$ glenoid bone loss and off-track Hill-Sachs lesions have been considered risk factors for recurrence. In patients with a glenoid bone defect more than 20\%-25\%, a bone graft procedure, such as the Latarjet procedure, is preferred regardless of the glenoid track concept, because compared with arthroscopic stabilization procedure, it provides greater postoperative stability. For patients with a borderline glenoid bone defect (around 20\%), surgeons should discuss surgical options with the patients, considering their demand and physical activity level. In addition, the surgeon should take care to prevent postoperative instability and long-term complications. Arthroscopic soft-tissue reconstruction including labral repair and capsular plication combined with the additional remplissage procedure is an anatomical procedure and could be considered as one of the primary treatment methods for patients with glenoid bone defects around $20 \%$. Therefore, treatment strategies for recurrent anterior shoulder instability combined with bone defects should be determined more flexibly on the basis of the patient's individual condition.

Keywords: Shoulder, Joint instability, Glenoid cavity, Humeral head, Bankart lesions

In the treatment of recurrent anterior instability of the shoulder, surgeons frequently encounter bipolar bone defects-glenoid bone defects combined with humeral bone defects. ${ }^{1)}$ The reported incidence of bipolar bone defects in patients with fewer than five dislocation events is $44 \%$; however, the incidence increases up to $82 \%$ with the increase in the number of dislocations. ${ }^{11}$ Bipolar bone defects are known as one of the most important risk factors for postoperative recurrence of anterior shoulder instability and surgical failure. ${ }^{2-4)}$ Therefore, it is important to determine optimal surgical procedures by preoperatively assess-

Received April 24, 2019; Accepted August 26, 2019

Correspondence to: Sang-Jin Shin, MD

Department of Orthopedic Surgery, Ewha Womans University Seoul Hospital, Ewha Womans University School of Medicine, 260 Gonghangdaero, Gangseo-gu, Seoul 07804, Korea

Tel: +82-2-2650-5143, Fax: +82-2-2642-0349

E-mail: sjshin622@ewha.ac.kr ing the size of a bipolar bone defect. Generally, a bipolar bone defect is considered substantial and increases the risk of recurrence if a glenoid bone defect is greater than $20 \%$ $25 \%$ and an off-track Hill-Sachs lesion is present. ${ }^{2,3,5,6)}$ In 2000, Burkhart and De Beer ${ }^{2}$ reported a recurrence rate of $67 \%$ after arthroscopic Bankart repair in patients with an inverted pear-shaped glenoid. They recommended bony procedures such as the Latarjet procedure to improve postoperative stability in patients with large glenoid bone defects. However, recent studies have demonstrated satisfactory clinical outcomes in patients with large bone defects after arthroscopic Bankart repair using additional procedures such as the remplissage procedure or capsular plication. ${ }^{7-10)}$ Still, the $20 \%-25 \%$ glenoid bone defect is often considered the critical cutoff in the literature. In this review article, the authors provide an overview of the effect of glenoid and humeral bone defects on shoulder instability and treatment strategies for recurrent anterior instability of the shoulder with bone defects. 
Park et al. Effects of Bone Defects on Recurrent Anterior Instability

Clinics in Orthopedic Surgery • Vol. 12, No. 2, $2020 \bullet$ www.ecios.org

\section{EFFECT OF GLENOID BONE DEFECTS}

Glenoid bone defects are known as one of the important risk factors for recurrence after surgical treatment of instability. ${ }^{2,4)}$ The size of a glenoid bone defect should be preoperatively estimated to determine the optimal surgical procedure for recurrent shoulder instability. On the basis of the defect size, surgeons choose either an arthroscopic soft-tissue stabilization procedure or a bone graft procedure. ${ }^{3)}$ However, the cutoff value for determining the surgical procedure remains controversial. The general consensus is that greater than $20 \%-25 \%$ glenoid bone loss requires a bony procedure such as the Latarjet procedure. ${ }^{2,3,11)}$ Bigliani et al. ${ }^{12)}$ first emphasized the importance of bone defects that affect more than $25 \%$ of the glenoid width in the choice of a treatment method for recurrent anterior shoulder instability; they recommended bony reconstruction procedures as primary surgical treatment. In a recent clinical study including 223 patients who underwent arthroscopic Bankart repair, the postoperative recurrence rate increased up to $21 \%$ when the glenoid defect was more than $20 \%$ of the glenoid width. ${ }^{13)}$ However, some recent studies have reported that the critical cutoff value should be lower than $20 \% .{ }^{14-16)}$ Shaha et al. ${ }^{14)}$ evaluated clinical outcomes and recurrence rates among 72 patients with anterior shoulder instability divided according to the extent of bone defects. They suggested that a glenoid bone defect above $13.5 \%$ led to a clinically significant decrease in the Western Ontario Shoulder Instability score even in patients without postoperative recurrence. Another clinical study also proposed that the cutoff value of glenoid bone defects for surgical failure should be $17.3 \%$ based on the assessment of 169 patients with anterior glenoid erosion. ${ }^{15)}$ In the study, compared to patients with a bone defect of less than $17.3 \%$, patients with a glenoid bone defect of more than $17.3 \%$ showed a significantly higher rate of surgical failure. These results were also supported biomechanically by cadaveric studies. In a biomechanical study, Shin et al. ${ }^{17)}$ simulated $10 \%, 15 \%, 20 \%$, and $25 \%$ glenoid bone defects with osteotomies. After Bankart repair of each shoulder, they found that shoulders with a glenoid bone defect of $15 \%$ or more had significantly higher anterior glenohumeral translation than shoulders with less glenoid loss. Therefore, even a glenoid bone defect less than $20 \%$ should be considered as a critical amount of bone defect that requires surgical procedures.

\section{EFFECT OF HUMERAL BONE DEFECTS}

Humeral bone defects are frequently accompanied by glenoid bone defects especially in patients who undergo recurrent instability events. ${ }^{1)}$ Because of their interaction during shoulder abduction and external rotational movement, it is important to address these two lesions simultaneously. The glenoid track concept is an excellent tool to evaluate bipolar bone defects and predict postoperative recurrence by using three-dimensional computed tomography (3D CT) or magnetic resonance imaging. ${ }^{5,18-20)}$ Metzger et al. ${ }^{21)}$ showed that the glenoid track concept is a good preoperative predictor of humeral head engagement and may help to guide surgical decision making. They classified patients on the basis of the glenoid track concept and then compared with clinical evidence of humeral head engagement found during arthroscopic examination. Among patients with an off-track lesion, $84.5 \%$ had clinical evidence of engagement, whereas only $12.4 \%$ of patients with an on-track lesion showed clinical engagement. Many studies regarding the glenoid track concept demonstrated that the off-track lesion is a strong predictor of postoperative recurrence. One clinical study showed a $75 \%$ recurrence rate in eight patients with off-track lesions and only an $8 \%$ recurrence rate in 49 patients with ontrack lesions. ${ }^{6}$ The positive predictive value of off-track measurement for postoperative recurrence was $75 \%$-the value was more than $20 \%$ higher than that of glenoid bone defects (44\%), although the sample size of that study was relatively small. In another study evaluating 100 patients who underwent an arthroscopic stabilization procedure, the postoperative recurrence rate was significantly higher in patients with off-track lesions (33\%) than in patients with on-track lesions (6\%). ${ }^{22)}$ Therefore, Di Giacomo et al. ${ }^{3)}$ recommended arthroscopic Bankart repair with additional remplissage procedure in patients with off-track lesions if glenoid bone loss was less than $20 \%$. Park et al. ${ }^{22)}$ evaluated clinical outcomes of 23 patients with off-track lesions after arthroscopic Bankart repair combined with additional remplissage procedure. ${ }^{23)}$ They performed the additional remplissage procedure only in patients with an engaging humeral head in arthroscopic examination after Bankart repair with capsular plication. As a result, compared with patients with on-track lesions, patients with off-track lesions showed satisfactory clinical outcomes and recurrence rates after arthroscopic Bankart repair with the remplissage procedure. They emphasized the greater importance of the glenoid bone defect than the off-track lesion as a predictor of postoperative surgical failure.

There would be some weaknesses of the glenoid track concept, because on- or off-track lesions are determined only by the bone defect size of the humeral head and glenoid although soft-tissue conditions such as cap- 
Park et al. Effects of Bone Defects on Recurrent Anterior Instability

Clinics in Orthopedic Surgery • Vol. 12, No. 2, $2020 \bullet$ www.ecios.org

sulolabrum and glenohumeral ligaments are also important factors for postoperative stability. ${ }^{9,10)}$ In patients with high-quality anterior capsule, the anterior capsule usually becomes tight and the humeral head rotates internally after Bankart repair with capsular plication. The location of the Hill-Sachs lesion is then altered far from the glenoid anterior margin, leaving little chance to reach the glenoid anterior margin. On the other hand, in patients with poorquality anterior capsule, this phenomenon would not occur even after proper capsular plication. The shape of HillSachs lesions would also affect the glenoid track concept. In patients with a wide and shallow Hill-Sachs lesion, there would be little possibility of humeral head engagement, even if it is an off-track lesion by the glenoid track concept. $^{24)}$

\section{TREATMENT STRATEGY FOR RECURRENT ANTERIOR INSTABILITY WITH BONE DEFECTS}

The size of a glenoid bone defect could be calculated on the en face view of preoperative 3D CT by using the Sugaya method. ${ }^{25)}$ Surgeons should determine whether a bony procedure is needed on the basis of the size of a glenoid bone defect. The critical value of a glenoid defect size has been widely considered as $20 \%-25 \%$; however, that value should be lowered to $13 \%-17 \%$ according to recent clinical and biomechanical studies. ${ }^{14-17)}$ In the treatment of patients with borderline glenoid bone defects, surgeons should discuss surgical options with patients because compared with arthroscopic Bankart repair, bony procedures have inherent advantages and disadvantages. In a recent study comparing clinical outcomes after the Latarjet procedure and arthroscopic Bankart repair in patients with a borderline glenoid bone loss of $15 \%-20 \%$, both procedures
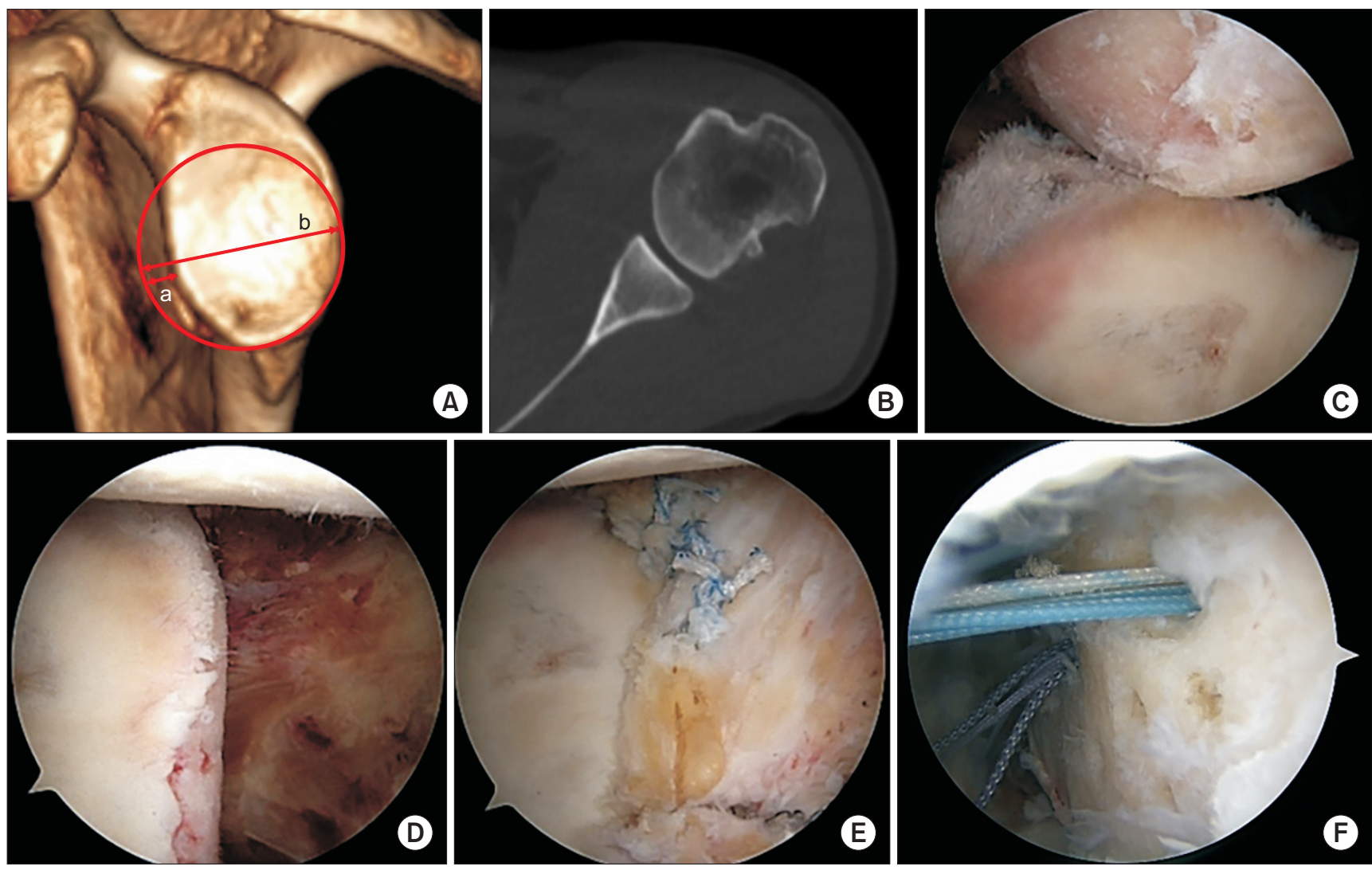

Fig. 1. A 38-year-old male with recurrent anterior instability of the left shoulder. All arthroscopic images were taken from an anterosuperior portal. (A) The glenoid defect size was calculated as $16.3 \%$ (a) of the widest glenoid width (b) on the en face view of three-dimensional computed tomography (CT). (B) The wide Hill-Sachs lesion was observed on the axial view of CT. (C) The Hill-Sachs lesion was engaging the anterior margin of the glenoid in the anterior apprehension position (the arm in $90^{\circ}$ abduction and $90^{\circ}$ external rotation). (D) The anterior capsuloligamentous complex was contracted and medially retracted without appropriate tension. (E) The capsuloligamentous complex regained appropriate tension after mobilization and repair using suture anchors. (F) Additional remplissage procedure using two suture anchors was performed because of the engaging Hill-Sachs lesion even after Bankart repair. 
Park et al. Effects of Bone Defects on Recurrent Anterior Instability

Clinics in Orthopedic Surgery • Vol. 12, No. 2, $2020 \bullet$ www.ecios.org

provided satisfactory clinical outcomes and pain relief; however, the recurrence rate was significantly lower after the Latarjet procedure than arthroscopic Bankart repair. ${ }^{26)}$ For patients with more than $20 \%-25 \%$ glenoid bone loss, many clinical studies recommend bony procedures such as the Latarjet procedure because of the low recurrence rate: $5 \%-17 \%$ after the Latarjet procedure vs $11 \%-16 \%$ after the arthroscopic stabilization procedure. ${ }^{7,8,27,28)}$ According to a meta-analysis study comparing clinical outcomes and recurrence rates after the Latarjet procedure and Bankart repair, the Latarjet procedure offered greater postoperative stability although differences in the glenoid bone defect size were not considered in the study. However, compared with arthroscopic stabilization surgery, the bony procedure has been associated with a higher incidence of surgical complications. One meta-analysis reported a $13.4 \%$ overall complication rate after glenoid bone grafting, such as iatrogenic nerve palsy, graft nonunion, fracture, hematoma formation, and screw loosening. ${ }^{29)}$ Compared to the Latarjet procedure, arthroscopic Bankart repair showed a lower incidence of postoperative complications $(0 \%-3.1 \%)$ even when combined with a complex bone incorporation procedure. ${ }^{30,31)}$ Therefore, patient's demand and physical activity level should be considered first when the surgeon determines surgical options in patients with a glenoid defect around 20\%. A recent clinical analysis provided satisfactory clinical outcomes after an arthroscopic stabilization procedure combined with the remplissage procedure, which is considered as a primary treatment option even in patients with a glenoid bone defect more than $20 \% .{ }^{11)}$ The authors of the study proposed that compared to nonanatomic reconstruction such as bony procedures, anatomical reconstruction using soft-tissue procedures including labral repair with capsular plication would result in less complications.

For the Hill-Sachs lesion, the engagement into the anterior edge of the glenoid was the important factor for determining the necessity of additional procedures such as the remplissage procedure in the past. Nowadays, treatment of the Hill-Sachs lesion is based on the combined glenoid defect. It would be important to determine whether the patient has an on- or off-track lesion. According to the glenoid track concept, the width of the glenoid track and the width of the Hill-Sachs lesion are used to determine whether the patient has an on- or off-track lesion.
The width of the glenoid track is calculated as $83 \%$ of the normalized glenoid width minus the glenoid bone defect width. ${ }^{19)}$ If the width of the Hill-Sachs lesion is greater than the width of the glenoid track, the patient is considered to have an off-track lesion, whereas an on-track lesion is considered present in the opposite case. Generally, in patients with recurrent instability who have an on-track lesion and less than $20 \%$ glenoid bone loss, isolated Bankart repair is recommended; the additional remplissage procedure is recommended in patients with an off-track lesion and less than 20\% glenoid bone loss. ${ }^{3)}$ Bone graft procedures are necessary in patients who have a glenoid bone defect of more than $20 \%$ regardless of the glenoid track lesion. This treatment algorithm was supported by a recent study comparing clinical outcomes after arthroscopic Bankart repair with selective remplissage procedure between patients with and without off-track lesions. ${ }^{23)}$ In the study, patients with an off-track lesion showed clinical outcomes and recurrence rates comparable to those in patients with an ontrack lesion after arthroscopic Bankart repair with selective remplissage procedure. Selective remplissage procedure, performed only when engagement of the humeral head is observed in arthroscopic examination after arthroscopic Bankart repair with capsular plication, should be also considered as a treatment option for off-track lesions (Fig. 1).

\section{CONCLUSION}

In the treatment of recurrent anterior instability of the shoulder, the proper treatment strategy is determined on the basis of the evaluation of glenoid and humeral bone defects. However, there are various measurement methods, and the critical value of glenoid and humeral bone defects to determine surgical procedures has yet to be established. Therefore, the treatment strategy for recurrent anterior instability of the shoulder should be determined more flexibly by the patient's condition. For more individualized treatment, surgeons should discuss surgical options with patients, considering their demand and physical activity level.

\section{CONFLICT OF INTEREST}

No potential conflict of interest relevant to this article was reported.

\section{REFERENCES}


Park et al. Effects of Bone Defects on Recurrent Anterior Instability

Clinics in Orthopedic Surgery • Vol. 12, No. 2, $2020 \bullet$ www.ecios.org

with traumatic anterior instability. Am J Sports Med. 2015; 43(11):2763-73.

2. Burkhart SS, De Beer JF. Traumatic glenohumeral bone defects and their relationship to failure of arthroscopic Bankart repairs: significance of the inverted-pear glenoid and the humeral engaging Hill-Sachs lesion. Arthroscopy. 2000; 16(7):677-94.

3. Di Giacomo G, Itoi E, Burkhart SS. Evolving concept of bipolar bone loss and the Hill-Sachs lesion: from "engaging/ non-engaging" lesion to "on-track/off-track" lesion. Arthroscopy. 2014;30(1):90-8.

4. Nakagawa S, Mae T, Sato S, Okimura S, Kuroda M. Risk factors for the postoperative recurrence of instability after arthroscopic Bankart repair in athletes. Orthop J Sports Med. 2017;5(9):2325967117726494.

5. Itoi E. 'On-track' and 'off-track' shoulder lesions. EFORT Open Rev. 2017;2(8):343-51.

6. Shaha JS, Cook JB, Rowles DJ, Bottoni CR, Shaha SH, Tokish JM. Clinical validation of the glenoid track concept in anterior glenohumeral instability. J Bone Joint Surg Am. 2016;98(22):1918-23.

7. Kim SJ, Kim SH, Park BK, Chun YM. Arthroscopic stabilization for recurrent shoulder instability with moderate glenoid bone defect in patients with moderate to low functional demand. Arthroscopy. 2014;30(8):921-7.

8. Mologne TS, Provencher MT, Menzel KA, Vachon TA, Dewing CB. Arthroscopic stabilization in patients with an inverted pear glenoid: results in patients with bone loss of the anterior glenoid. Am J Sports Med. 2007;35(8):1276-83.

9. Ozbaydar M, Elhassan B, Diller D, Massimini D, Higgins LD, Warner JJ. Results of arthroscopic capsulolabral repair: Bankart lesion versus anterior labroligamentous periosteal sleeve avulsion lesion. Arthroscopy. 2008;24(11):1277-83.

10. Shin SJ, Ko YW, Lee J. Intra-articular lesions and their relation to arthroscopic stabilization failure in young patients with first-time and recurrent shoulder dislocations. J Shoulder Elbow Surg. 2016;25(11):1756-63.

11. Park I, Park CJ, Lee JH, Hyun HS, Park JY, Shin SJ. Clinical outcomes and recurrence rates after arthroscopic stabilization procedures in young patients with a glenoid bone erosion: a comparative study between glenoid erosion more and less than 20. Arthroscopy. 2018;34(8):2287-93.

12. Bigliani LU, Newton PM, Steinmann SP, Connor PM, Mcllveen SJ. Glenoid rim lesions associated with recurrent anterior dislocation of the shoulder. Am J Sports Med. 1998; 26(1):41-5.

13. Park I, Lee JH, Hyun HS, Oh MJ, Shin SJ. Effects of bone incorporation after arthroscopic stabilization surgery for bony
Bankart lesion based on preoperative glenoid defect size. Am J Sports Med. 2018;46(9):2177-84.

14. Shaha JS, Cook JB, Song DJ, et al. Redefining "critical" bone loss in shoulder instability: functional outcomes worsen with "subcritical" bone loss. Am J Sports Med. 2015;43(7): 1719-25.

15. Shin SJ, Kim RG, Jeon YS, Kwon TH. Critical value of anterior glenoid bone loss that leads to recurrent glenohumeral instability after arthroscopic bankart repair. Am J Sports Med. 2017;45(9):1975-81.

16. Yamamoto A, Massimini DF, DiStefano J, Higgins LD. Glenohumeral contact pressure with simulated anterior labral and osseous defects in cadaveric shoulders before and after soft tissue repair. Am J Sports Med. 2014;42(8):1947-54.

17. Shin SJ, Koh YW, Bui C, et al. What is the critical value of glenoid bone loss at which soft tissue bankart repair does not restore glenohumeral translation, restricts range of motion, and leads to abnormal humeral head position? Am J Sports Med. 2016;44(11):2784-91.

18. Momaya AM, Tokish JM. Applying the glenoid track concept in the management of patients with anterior shoulder instability. Curr Rev Musculoskelet Med. 2017;10(4):463-8.

19. Omori Y, Yamamoto N, Koishi H, et al. Measurement of the glenoid track in vivo as investigated by 3-dimensional motion analysis using open MRI. Am J Sports Med. 2014;42(6): 1290-5.

20. Trivedi S, Pomerantz ML, Gross D, Golijanan P, Provencher MT. Shoulder instability in the setting of bipolar (glenoid and humeral head) bone loss: the glenoid track concept. Clin Orthop Relat Res. 2014;472(8):2352-62.

21. Metzger PD, Barlow B, Leonardelli D, Peace W, Solomon DJ, Provencher MT. Clinical application of the "glenoid track" concept for defining humeral head engagement in anterior shoulder instability: a preliminary report. Orthop J Sports Med. 2013;1(2):2325967113496213.

22. Park I, Kang JS, Jo YG, Kim SW, Shin SJ. Off-track HillSachs lesions do not increase postoperative recurrent instability after arthroscopic Bankart repair with selective Remplissage procedure. Knee Surg Sports Traumatol Arthrosc. 2019;27(12):3864-70.

23. Kurokawa D, Yamamoto N, Nagamoto H, et al. The prevalence of a large Hill-Sachs lesion that needs to be treated. J Shoulder Elbow Surg. 2013;22(9):1285-9.

24. Sugaya H, Moriishi J, Dohi M, Kon Y, Tsuchiya A. Glenoid rim morphology in recurrent anterior glenohumeral instability. J Bone Joint Surg Am. 2003;85(5):878-84.

25. Jeon YS, Jeong HY, Lee DK, Rhee YG. Borderline glenoid bone defect in anterior shoulder instability: latarjet proce- 
Park et al. Effects of Bone Defects on Recurrent Anterior Instability

Clinics in Orthopedic Surgery • Vol. 12, No. 2, $2020 \bullet$ www.ecios.org

dure versus bankart repair. Am J Sports Med. 2018;46(9): 2170-6.

26. Mizuno N, Denard PJ, Raiss P, Melis B, Walch G. Long-term results of the Latarjet procedure for anterior instability of the shoulder. J Shoulder Elbow Surg. 2014;23(11):1691-9.

27. Yang JS, Mazzocca AD, Cote MP, Edgar CM, Arciero RA. Recurrent anterior shoulder instability with combined bone loss: treatment and results with the modified latarjet procedure. Am J Sports Med. 2016;44(4):922-32.

28. Beran MC, Donaldson CT, Bishop JY. Treatment of chronic glenoid defects in the setting of recurrent anterior shoulder instability: a systematic review. J Shoulder Elbow Surg. 2010; 19(5):769-80.
29. An VV, Sivakumar BS, Phan K, Trantalis J. A systematic review and meta-analysis of clinical and patient-reported outcomes following two procedures for recurrent traumatic anterior instability of the shoulder: Latarjet procedure vs. Bankart repair. J Shoulder Elbow Surg. 2016;25(5):853-63.

30. Sugaya H, Moriishi J, Kanisawa I, Tsuchiya A. Arthroscopic osseous Bankart repair for chronic recurrent traumatic anterior glenohumeral instability. J Bone Joint Surg Am. 2005; 87(8):1752-60.

31. Sugaya H, Moriishi J, Kanisawa I, Tsuchiya A. Arthroscopic osseous Bankart repair for chronic recurrent traumatic anterior glenohumeral instability. J Bone Joint Surg Am. 2005; 87(8):1752-60. 